hep-th/0611094

\title{
Dark Energy From Bulk Matter
}

\author{
C. Bogdanos, A. Dimitriadis and K. Tamvakis \\ Physics Department, University of Ioannina \\ Ioannina GR451 10, Greece
}

\begin{abstract}
We consider the possibility of getting accelerated expansion and $w=-1$ crossing in the context of a braneworld cosmological setup, endowed with a bulk energy-momentum tensor. For a given ansatz of the bulk content, we demonstrate that the bulk pressures dominate the dynamics at late times and can lead to accelerated expansion. We also analyze the constraints under which we can get a realistic profile for the effective equation of state and conclude that matter in the bulk has the effect of dark energy on the brane. Furthermore, we show that it is possible to simulate the behavior of a Chaplygin gas using non-exotic bulk matter.
\end{abstract}


Recent experimental results suggesting an accelerating expansion of the universe [1], [2], have become the subject of active research. During the last few years, a number of theories were proposed to answer the question of the origin of such an acceleration. The most common explanation is to assume the existence of some, for the moment unknown, "dark energy" component, which drives the expansion. The physics of this dark energy is still speculative, as no direct experiment can provide us with actual information about its nature. We can only indirectly probe its properties by studying the dynamics of cosmic expansion. There are several theories which attempt to describe dark energy. Among them are the phantom [3] and quintessence [4] theories, quiessence (cosmological constant) and other forms of exotic matter [5], like the Chaplygin gas [6] (see also [7] for a review and a more complete list of references). In these theories, dark energy is attributed to the presence of new fields with non-trivially modified equations of state.

One can follow a different route and try instead modified theories of gravity. These are theories which extend or modify GR in such a way, so that dark energy is not some new -maybe exotic- matter constituent, but merely a manifestation of the modified dynamics of geometry. In this category we find scalar-tensor theories of gravity, the socalled modified gravity models and theories with extra dimensions [8], [9]. Braneworld models, where our four-dimensional world is realized as a brane embedded in a higher dimensional space (bulk), have received considerable attention mainly due to the hope of tackling hierarchy problems and explaining the large disparity between the Planck and the electroweak scale [10]. Brane models like these are also directly connected to String Theory. An interesting consequence of brane models is that they allow for the presence of five-dimensional matter which propagates in the bulk space and may interact with the matter content of the braneworld [12]. Such an interaction can alter the profile of the cosmic expansion and lead to a behavior that would resemble that of dark energy.

It has already been shown [13] that a configuration of bulk matter resembling a perfect fluid moving along the fifth dimension is capable of producing accelerated expansion and give an effective equation of state which can cross the $w=-1$ line. However, for the later to occur, the existence of a large and negative dark radiation term was needed. The presence of such a term may cause problems in early times, as it could lead to a negative effective energy density (although the validity of the modified Friedmann equation for large redshifts is in question). Our goal here is to replicate these effects by ignoring the dark radiation term and considering only bulk matter as the late time dominant term. We are also interested in determining not only under which conditions we can have $w_{\text {eff }}<-1$, but whether we can obtain a temporal profile of the crossing which is at least qualitatively in accordance with observations for $0 \leq z \leq 1$. As we will see, these requirements can be met, provided we have a specific ansatz for bulk matter and the parameters are properly set. We will also demonstrate the equivalence of this bulk matter to a Chaplygin gas.

We will start by considering a braneworld cosmological model, with a 5D bulk space and a positive tension brane embedded in it. The action of the model is

$$
\mathcal{S}=\int d^{5} x \sqrt{-G}\left(2 M^{3} R-\Lambda+\mathcal{L}_{B}^{(m)}\right)+\int d^{4} x \sqrt{-g}\left(-\sigma+\mathcal{L}_{b}^{(m)}\right) .
$$

The 5D metric is $G_{M N}$ with signature $(-,+,+,+,+)$, while $g_{\mu \nu}$ is the induced metric on the brane and $\mathrm{M}$ denotes the five-dimensional Planck mass. We shall assume that the metric can be written as

$$
d s^{2}=-n^{2}(y, t) d t^{2}+a^{2}(y, t) \gamma_{i j} d x^{i} d x^{j}+b^{2}(y, t) d y^{2},
$$


where $y$ denotes the fifth dimension (the brane is situated at $y=0$ ) and $\gamma_{i j}$ is the metric of a maximally symmetric three-space.

Varying the action with respect to the metric, we obtain Einstein's equations

$$
\mathcal{G}_{M N} \equiv R_{M N}-\frac{1}{2} G_{M N} R=\frac{1}{4 M^{3}} T_{M N}
$$

The energy-momentum tensor $T_{M N}$ resulting from the above Action is of the form

$$
T_{M N}=T_{M N}^{(B)}+T_{M N}^{(b)}-G_{M N} \Lambda-g_{\mu \nu} \sigma \delta(y) \delta_{M}^{\mu} \delta_{N}^{\nu},
$$

where $T_{M N}^{(B)}$ results from $\mathcal{L}_{B}^{(m)}$ and $T_{M N}^{(b)}$ results from $\mathcal{L}_{b}^{(m)}$. In particular, we will assume an energy-momentum tensor for the bulk content of the form

$$
T_{N}^{(B)^{M}}=\left(\begin{array}{ccc}
-\rho_{B} & 0 & P_{5} \\
0 & P_{B} \delta^{i}{ }_{j} & 0 \\
-\frac{n^{2}}{b^{2}} P_{5} & 0 & \bar{P}_{B}
\end{array}\right), T_{M N}^{(B)}=\left(\begin{array}{ccc}
\rho_{B} n^{2} & 0 & -n^{2} P_{5} \\
0 & P_{B} a^{2} \gamma_{i j} & 0 \\
-n^{2} P_{5} & 0 & \bar{P}_{B} b^{2}
\end{array}\right)
$$

The quantities which are of interest here are the pressures $\bar{P}_{B}$ and $P_{5}$, as these two enter the cosmological equations of motion. $P_{5}$ is the term responsible for energy exchange between the brane and the bulk. The corresponding energy-momentum tensor for the brane matter is

$$
T_{N}^{(b)}{ }_{N}^{M}=\frac{\delta(y)}{b} \operatorname{diag}(-\rho, p, p, p, 0) .
$$

Substituting the above ansatze for the metric (2) and for the energy-momentum tensor (5), (6) into the equations of motion (3), we can obtain the set of cosmological equations for the components of the metric [11][12][13]. These equations simplify if we choose Gauss normal coordinates, such that $b(t, y)=1$. We can also make use of the freedom to take $n(0, t)=n_{0}(t)=1$. After these simplifications, we get for the evolution equations 1 on the brane $\left(a_{0}(t) \equiv a(0, t)\right)$

$$
\begin{gathered}
\dot{\rho}+3(\rho+p) \frac{\dot{a}_{0}}{a_{0}}=-2 P_{5}, \\
\frac{\ddot{a}_{0}}{a_{0}}+\left(\frac{\dot{a}_{0}}{a_{0}}\right)^{2}+\frac{k}{a_{0}^{2}}=\frac{1}{\left(24 M^{3}\right)^{2}}(\sigma+\rho)(2 \sigma-\rho-3 p)+\frac{1}{12 M^{3}}\left(\Lambda-\bar{P}_{B}\right) .
\end{gathered}
$$

The first expresses energy conservation on the brane. We see that positive $P_{5}$ means energy outflow from the brane, while for $P_{5}$ negative we get the opposite effect, with energy flowing from the bulk into the brane. The second equation is the Friedmann equation for the brane world. The " 55 " component of the bulk pressure appears on the right hand side of this equation (8) affecting the cosmological evolution.

We assume that matter on the brane has an equation of state $\rho=w p$. Introducing the parameters $\beta \equiv\left(24 M^{3}\right)^{-2}$ and $\gamma \equiv \sigma \beta$ and omitting the "o" subscript from the scale factor, we can rewrite the cosmological evolution equation (8) as

$$
\frac{\ddot{a}}{a}+\left(\frac{\dot{a}}{a}\right)^{2}+\frac{k}{a^{2}}=\gamma \rho(1-3 w)-\beta \rho^{2}(1+3 w)-\frac{\bar{P}_{B}}{12 M^{3}}+\frac{\lambda}{12 M^{3}} .
$$

\footnotetext{
${ }^{1} k=-1,0,1$ is the maximally symmetric internal space curvature parameter.
} 
We also assume the Randall-Sundrum fine-tuning to hold, so that the effective cosmological constant on the brane

$$
\lambda \equiv \Lambda+\frac{\sigma^{2}}{24 M^{3}}
$$

is equal to zero, i.e. $\lambda=0$. The cosmological evolution equation (9) can then be expressed as a set of two equations using a dark energy field $\chi$

$$
\begin{gathered}
\left(\frac{\dot{a}}{a}\right)^{2}=\beta \rho^{2}+2 \gamma \rho-\frac{k}{a^{2}}+\chi-\frac{\bar{P}_{B}}{12 M^{3}}, \\
\dot{\chi}+4 \frac{\dot{a}}{a}\left(\chi-\frac{\bar{P}_{B}}{24 M^{3}}\right)=4 \beta\left(\rho+\frac{\gamma}{\beta}\right) P_{5}+\frac{\dot{\bar{P}}_{B}}{12 M^{3}},
\end{gathered}
$$

where the defining equation for $\chi$ is

$$
\frac{\ddot{a}}{a}=-\chi-(3 w+2) \beta \rho^{2}-(3 w+1) \gamma \rho .
$$

The first of these equations (11) is analogous to the Friedmann equation of standard cosmology. The dark energy field accounts for contributions to the Friedmann equation which do not come from ordinary brane matter. In the absence of bulk content, it is equal to a dark radiation term, a consequence of the presence of the extra dimension. When $\bar{P}_{B}$ or $P_{5}$ are non-zero, it also receives contributions from these terms. Thus the origin of $\chi$ can be both geometric and matter-related.

We are interested in scenarios where the energy density of the brane is much lower than its tension, that is $\rho<<\sigma$. We can then disregard terms quadratic in $\rho$ and, thus, we get the simplified cosmological equations

$$
\begin{gathered}
\left(\frac{\dot{a}}{a}\right)^{2}=2 \gamma \rho-\frac{k}{a^{2}}+\chi-\frac{\bar{P}_{B}}{12 M^{3}}, \\
\dot{\chi}+4 \frac{\dot{a}}{a}\left(\chi-\frac{\bar{P}_{B}}{24 M^{3}}\right)=4 \gamma P_{5}+\frac{\dot{\bar{P}}_{B}}{12 M^{3}} .
\end{gathered}
$$

and, of course, the continuity equation

$$
\dot{\rho}+3 \rho \frac{\dot{a}}{a}(1+w)=-2 P_{5} .
$$

The functions $\bar{P}_{B}, P_{5}$ are functions of time corresponding to the values of $\bar{P}_{B}(y, t)$ and $P_{5}(y, t)$ on the brane. The energy-momentum conservation $\nabla_{M} T_{N}^{M}=0$ cannot fully determine $\bar{P}_{B}$ and $P_{5}$ and a particular model of the bulk matter is required [13. Here we are going to consider a general ansatz for the bulk pressures (see also [19])

$$
\bar{P}_{B}=D a^{\nu}, \quad P_{5}=F\left(\frac{\dot{a}}{a}\right) a^{\mu} .
$$

where $F$ and $D$ are constant parameters. For this choice of pressures, the equation for $\chi$ can be solved exactly to give

$$
\chi=\frac{\mathcal{C}}{a^{4}}+2 \delta \frac{(\nu+2)}{(\nu+4)} a^{\nu}+\frac{4 F \gamma}{(\mu+4)} a^{\mu},
$$


where we have defined $\delta \equiv D / 24 M^{3}$. Similarly, we can proceed with the integration of (16) and get

$$
\rho=\frac{\tilde{\mathcal{C}}}{a^{3(1+w)}}-\frac{2 F}{[3(1+w)+\mu]} a^{\mu} .
$$

Substituting (18) and (19) into the Friedmann equation (14), we can write it in a conventional form as

$$
\left(\frac{\dot{a}}{a}\right)^{2}+\frac{k}{a^{2}}=\frac{8 \pi}{3} G_{N} \rho_{e f f},
$$

where $G_{N}=3 \gamma / 4 \pi=3 \sigma / 4 \pi\left(24 M^{3}\right)^{2}$ is the $4 D$ Newton's constant and the effective energy density $\rho_{\text {eff }}$ stands for

$$
\rho_{e f f}=\frac{\tilde{\mathcal{C}}}{a^{3(1+w)}}+\frac{\mathcal{C} / 2 \gamma}{a^{4}}-\frac{2 \delta}{\gamma(\nu+4)} a^{\nu}+\frac{2(3 w-1) F}{(\mu+4)[3(1+w)+\mu]} a^{\mu} .
$$

At late times, the term with the highest power in $a$ will dominate and we will get a scale factor $a(t) \sim t^{-\frac{2}{\max (\mu, \nu)}}$. Acceleration occurs whenever $\max (\mu, \nu)>-2$.

Using the already mentioned effective energy density, we can derive an expression for the effective equation of state parameter using the prescription 20]

$$
w_{e f f}^{(D)}=-1-\frac{1}{3} \frac{d \ln \left(\delta H^{2}\right)}{d \ln a},
$$

where $\delta H^{2}=H^{2} / H_{0}^{2}-\Omega_{m} a^{-3}$ accounts for all terms in the Friedmann equation not related to the brane matter, for which we have taken $w=0$. Using as our variable the redshift parameter $z=\frac{a_{0}}{a(t)}-1, w_{\text {eff }}$ has the general form

$$
w_{e f f}=-1+\frac{1}{3}\left(\frac{\nu A+\mu B(z+1)^{\nu-\mu}}{-A-B(z+1)^{\nu-\mu}}\right)
$$

where the parameters $A$ and $B$ depend on the bulk pressures

$$
A \equiv \frac{2 \delta}{\gamma(\nu+4)}=\frac{48 D M^{3}}{(\nu+4) \sigma}, \quad B \equiv \frac{2 F}{(\mu+3)(\mu+4)}
$$

We have assumed that the dark radiation parameter $\mathcal{C}$ is small and that, at late times, the dark radiation term $\sim \frac{\mathcal{C} / 2 \gamma}{a^{4}}$ is negligible with respect to the contribution of the pressures $\bar{P}_{B}$ and $P_{5}$. Thus, we have ignored this term in our expression for $w_{\text {eff }}$.

Equation (23) allows us to examine under which conditions we can have an effective equation of state which can exhibit $w_{\text {eff }}=-1$ crossing, as is suggested by recent observational data. In particular, we want to investigate what are the requirements that our model's parameters have to comply with, in order to get a qualitatively correct behavior for the evolution of $w_{\text {eff }}$ at late times. Current observational findings indicate that at the moment $(z=0)$ we are at a stage of accelerated expansion with $|q|$ of order unity and effective equation of state $w_{\text {eff }}=-1.21$. The $w_{\text {eff }}=-1$ crossing seems to have occurred at redshifts $z=0.2$. Therefore $w_{\text {eff }}$ seems to increase with $z$, starting with a value of about -1.21 at $z=0$ and crossing the $w_{\text {eff }}=-1$ line about $z=0.2$. Our goal is to obtain constraints on the values of our parameters $A, B, \mu$ and $\nu$ and see for which combinations we can have a $w_{\text {eff }}$ with the desired temporal profile, while having 
accelerated expansion. The corresponding late time deceleration parameter (where we have neglected the contribution from brane matter as well as the dark radiation term) is

$$
q \equiv-\frac{1}{H^{2}} \frac{\ddot{a}}{a}=-\frac{(\nu+2) A+(\mu+2) B(z+1)^{\nu-\mu}}{2 A+2 B(z+1)^{\nu-\mu}} .
$$

By inspecting equation (23), we first notice that the denominator is the effective energy density at late times and thus it has to be positive at the interval of $z$ we are interested in (in this discussion we focus on $0 \leq z \leq 1$ ). At $z=0$ this condition reduces to $A+B<0$. It is, thus, obvious that $A$ and $B$ cannot be both positive. Taking the derivative of (23), we obtain

$$
\frac{d w_{e f f}}{d z}=A B \frac{(\nu-\mu)^{2}(z+1)^{\nu-\mu-1}}{\left(A+B(z+1)^{\nu-\mu}\right)^{2}} .
$$

All terms in the right hand side of (26) are positive in the interval $0 \leq z \leq 1$, except from the product $A B$. In order to have an increasing $w_{\text {eff }}, A$ and $B$ must have the same sign, i.e. they must both be negative $(A, B<0)$. Next, we want $w_{\text {eff }}$ to assume a value less than -1 at $z=0$. In order to achieve this we must have $\nu A+\mu B<0$. There are four possible combinations:

1) $\mu, \nu>0$ : The constraint is satisfied.

2) $\mu>0, \nu<0: \nu A+\mu B<0$ when $\frac{\nu}{\mu}>-\frac{B}{A}$.

3) $\mu<0, \nu>0: \nu A+\mu B<0$ when $\frac{\nu}{\mu}<-\frac{B}{A}$.

4) $\mu, \nu<0$ : The constraint cannot be satisfied for $A$ and $B$ negative.

So, the first three cases seem to be allowed. However, a further constraint comes from the fact that we want $w_{\text {eff }}=-1$ for $z=0.2$. This reduces to the relation

$$
\frac{\nu}{\mu}=-\frac{B}{A}(1.2)^{\nu-\mu}
$$

In the case (1), with both $\mu$ and $\nu$ positive, the left hand side of (27) is positive, while the right hand side is negative and thus it is ruled out. Cases (2) and (3) can satisfy this equation without providing any additional constraints for the powers $\mu$ and $\nu$. Thus we conclude that the only choices of parameters which are in accordance with the general profile for the evolution of $w_{\text {eff }}$ for $0 \leq z \leq 1$ are:

1) $A, B<0$ with $\mu>0, \nu<0$ and $\frac{\nu}{\mu}>-\frac{B}{A}$.

2) $A, B<0$ with $\mu<0, \nu>0$ and $\frac{\nu}{\mu}<-\frac{B}{A}$.

Since one of the powers has to be positive, the condition $\max (\mu, \nu)>-2$ is always satisfied and we also get the desired accelerated expansion for late times. We also see that the ratio of bulk pressure parameters $A$ and $B$ determines the ratio of the two powers. A large difference between the initial pressures $D$ and $F$ would result in an accordingly large disparity between $\mu$ and $\nu$. This is demonstrated in Figure 1, where we give a graph of the deceleration parameter $(q)$ and $w_{\text {eff }}$, for a set of values that satisfy the above constraints. For this choice, we see that there is an accelerated expansion at late times and $w_{\text {eff }}$ has a realistic temporal profile.

The requirement that $A$ and $B$ must be negative has a direct impact on the sign of parameters $D$ and $F$ and the corresponding behavior of the underlying bulk content. In 


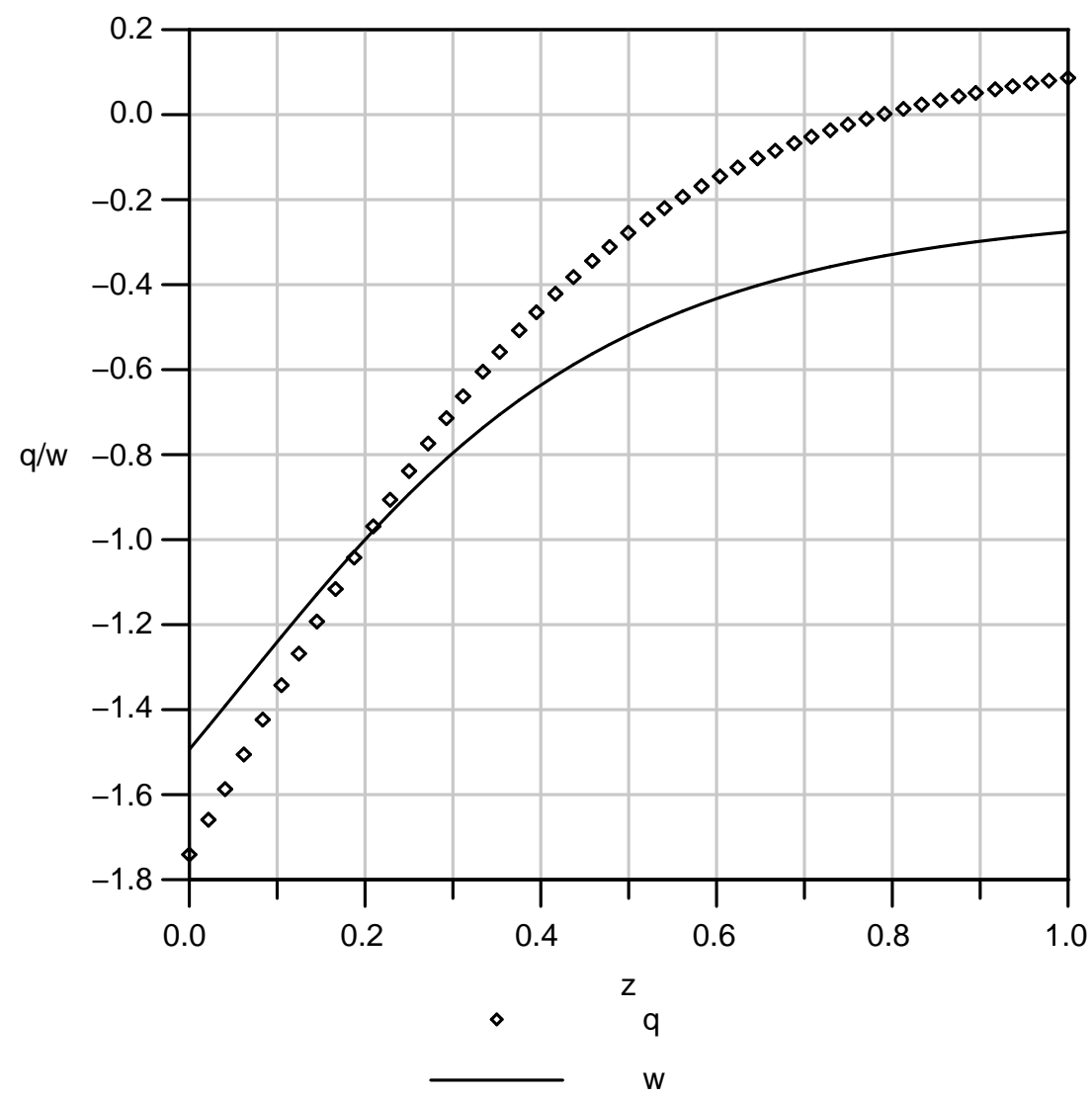

Figure 1: Graph of the deceleration parameter q and $w_{\text {eff }}$ for $A=-1, B=-2, \mu=2$, $\nu=-2$.

order to have $B<0$ with $\mu<-4$ or $\mu>-3$, we must have $P_{5}<0$, which means we have energy inflow from the bulk. When $-4<\mu<-3$, we get $P_{5}>0$ and energy outflow from the brane. Similarly, for $A<0$, we get $\bar{P}_{B}<0$ if $\nu>-4$. This means that the bulk content must have negative pressure. This resembles some sort of five-dimensional quintessence field (like a scalar field). For $\nu<-4$, the pressure $\bar{P}_{B}$ becomes positive and we can assume the bulk matter to behave as a regular relativistic fluid. Apparently, we cannot have both pressures positive, since then we would have two negative powers, which is excluded from the constraints obtained above. Notice also that we have managed to achieve $w_{\text {eff }}=-1$ crossing without the use of any bulk matter which violates energy conditions, like phantom fields. The presence of the dark radiation term was also not necessary to get the desired phenomenon, which manifests itself as a result of the interplay between the bulk pressure and the brane-bulk energy exchange terms. We, thus, have a description of dark energy in terms of a bulk energy-momentum tensor, which can drive accelerated expansion in the present era and provide a qualitatively correct picture for the evolution of the equation of state at late times.

As a further application of our model, we can show that with proper choice of parameters for the bulk pressure, we can simulate a Chaplygin gas, which is another candidate for quintessence. A Chaplygin gas is a perfect four-dimensional fluid which obeys the equation of state

$$
p=-\frac{A}{\rho}
$$


Assuming energy conservation in the form

$$
d\left(\rho a^{3}\right)=-p d\left(a^{3}\right),
$$

it is easy to show that for small $a$, i.e. early times, $\rho \propto a^{-3}$. This is the behavior we expect from dust matter with $w=0$. For large $a$ or late times, $\rho=-p$, which is the equation of state for cosmological constant. The Chaplygin gas behaves as dark matter at early times and as dark energy (cosmological constant) at late times and is a simple example of unified treatment of these two constituents. It is easy to see that the $w_{\text {eff }}$ in (23) can replicate this behavior and thus the bulk content can play a role similar to that of a Chaplygin gas. We first want the effective equation of state parameter to be equal to -1 at late times $(z=0)$. This can be satisfied for $\nu A+\mu B=0$, or

$$
\frac{\nu}{\mu}=-\frac{\mathrm{B}}{A}
$$

Additionally, we want $w_{\text {eff }}=0$ for $z>>1$. Depending on which is the highest power, we must have either $\mu=-3$ for $\nu-\mu>0$, or $\nu=-3$ for $\nu-\mu<0$. The corresponding conditions are then:

1) $\mu=-3, \nu=3 \frac{B}{A}$.

2) $\nu=-3, \mu=3 \frac{A}{B}$.

For the above choice of parameters, we can reproduce the effects of a Chaplygin gas using only bulk matter content.

Summarizing, we dealt with the problem of simulating the properties of dark energy in the context of brane world cosmology. We showed that by assuming the existence of an extra dimension and bulk matter, whose energy-momentum tensor is of the form (17), we can reproduce the observed accelerated expansion of the universe and the equation of state for the dark energy. We analyzed in detail the constraints our model parameters have to obey in order to get a realistic profile for the evolution of $w_{\text {eff }}$ for small $z$. The crossing of the $w_{\text {eff }}=-1$ line occurs naturally, without invoking exotic forms of bulk or brane matter that violate energy conditions. Our results also have the advantage of not depending on the dark radiation term and they consequently do not fix the sign of the $\mathcal{C}$ constant. We also showed that this model can reproduce predictions similar to those of other dark energy candidates, in particular the Chaplygin gas. We cannot, at the moment, fully justify the ansatz (17) in terms of a phenomenological bulk fluid. As it was shown in [13], this is due to the unknown spatial derivatives of the bulk pressures that appear in the equation of energy-momentum conservation for the bulk fluid. A specific model for the bulk matter is needed, in order to eliminate this extra freedom. Such a realistic model for the bulk matter, which may yield the desired behavior, is currently under investigation.

Acknowledgments. A. D. and C. B. wish to thank S. Nesseris for useful discussions. This research was co-funded by the European Union in the framework of the Program $\Pi Y \Theta A Г О Р А \Sigma-I I$ of the "Operational Program for Education and Initial Vocational Training" (EПEAEK) of the 3rd Community Support Framework of the Hellenic Ministry of Education, funded by $25 \%$ from national sources and by $75 \%$ from the European Social Fund (ESF). C. B. acknowledges also an Onassis Foundation fellowship. 


\section{References}

[1] A. G. Riess et al., Astron. J. 116, 1009 (1998); S. Perlmutter et al., Astrophys. J. 517, 565 (1999); A. G. Riess et al.Astrophys.J. 607, 665 (2004).

[2] D. N. Spergel, et al., WMAP Three Year Results: Implications for Cosmology, astro-ph/0603499.

[3] R. R. Calwell, Phys. Lett. B 545, 23 (2002); R. R. Caldwell, M. Kamionkowski and N. N. Weinberg, Phys. Rev. Lett. 91, 071301 (2003); J. M. Cline, S. Y. Jeon and G. D. Moore, Phys. Rev. D 70, 043543 (2004).

[4] R. R. Caldwell, R. Dave and P. J. Steinhardt, Phys. Rev. Lett. 80, 1582 (1998); P. J. E. Peebles and A. Vilenkin, Phys. Rev. D 59, 063505 (1999); P. J. Steinhardt, L. M. Wang and I. Zlatev, Phys. Rev. D 59, 123504 (1999); M. Doran and J. Jaeckel, Phys. Rev. D 66, 043519 (2002); A. R. Liddle, P. Parson and J. D. Barrow, Phys. Rev. D 50, 7222 (1994).

[5] H. Wei, R. G. Cai and D. F. Zeng, Class. Quant. Grav. 22, 3189 (2005) arXiv:hep-th/0501160.

[6] A. Y. Kamenshchik, U. Moschella and V. Pasquier, Phys. Lett. B 511 (2001) 265 arXiv:gr-qc/0103004.

[7] V. Sahni and A. Starobinsky, arXiv:astro-ph/0610026.

[8] N. Arkani-Hamed, S. Dimopoulos and G. R. Dvali, Phys. Lett. B 429 (1998) 263; I. Antoniadis, N. Arkani-Hamed, S. Dimopoulos and G. R. Dvali, Phys. Lett. B 436 257 (1998).

[9] G. R. Dvali, G. Gabadadze and M. Porrati, Phys. Lett. B 485, 208 (2000); G. R. Dvali, G. Gabadadze, M. Kolanovic and F. Nitti, Phys. Rev. D 64084004 (2001).

[10] L. Randall and R. Sundrum, Phys. Rev. Lett. 83 (1999) 3370; Phys. Rev. Lett. 83 (1999) 4690.

[11] P. Binetruy, C. Deffayet and D. Langlois, Nucl. Phys. B 565269 (2000); P. Binetruy, C. Deffayet, U. Ellwanger and D. Langlois, Phys. Lett. B 447285 (2000).

[12] E. Kiritsis, G. Kofinas, N. Tetradis, T. N. Tomaras and V. Zarikas, JHEP 0302 (2003) 035; E. Kiritsis, N. Tetradis and T. N. Tomaras, JHEP 0203 (2002) 019; P. S. Apostolopoulos and N. Tetradis, Phys. Rev. D 71043506 (2005); P. S. Apostolopoulos and N. Tetradis, Phys. Lett. B 633409 (2006); E. Kiritsis, JCAP 0510 014 (2005); K. I. Umezu, K. Ichiki, T. Kajino, G. J. Mathews, R. Nakamura and M. Yahiro, Phys. Rev. D 73063527 (2006).

[13] C. Bogdanos and K. Tamvakis, arXiv:hep-th/0609100.

[14] T. Padmanabhan, Phys. Rept. 380, 235 (2003); V. Sahni and A. A. Starobinsky, Int. J. Mod. Phys. D 9, 373 (2000); S. M. Carroll, Living Rev. Rel. 4, 1 (2001); S. Weinberg, Rev. Mod. Phys. 61, 1 (1989).

[15] I. Antoniadis, Phys. Lett. B 246, 377 (1990). 
[16] T. Shiromizu, K. Maeda and M. Sasaki, Phys. Rev. D 62 (2000) 024012.

[17] S. Pal, Phys. Rev. D 74 (2006) 024005 arXiv:gr-qc/0606085].

[18] S. Nesseris and L. Perivolaropoulos, Phys. Rev. D 70, 043531 (2004) [arXiv:astro-ph/0401556]; S. Nesseris and L. Perivolaropoulos, [arXiv:astro-ph/0610092].

[19] R.G. Cai, Y. Gong and B. Wang, JCAP 0603 (2006); P. S. Apostolopoulos and N. Tetradis, hep-th/0604014.

[20] E. V. Linder and A. Jenkins, Mon. Not. Roy. Astron. Soc. 346, 573 (2003). 